\title{
NON-RADIAL OSCILIATIONS IN ROTATING WHITE DWARFS
}

$$
\text { J. Papaloizou, J.E. Pringle }
$$

Institute of Astronomy, Madingley Rd., Cambridge CB3 OHA

In previous work (Papaloizou and Pringle, 1978), we investigated analytically the low frequency spectrum of a rotating pulsating star, taking the effects of rotation fully into account. In that case we found that not only are the g-modes substantially modified by the rotation, but also that a new set of modes appear (the $r$ modes) which are similar to Rossby waves in the Earth's atmosphere and oceans.

Here we present numerical computations of the oscillations of rapidly rotating white dwarfs. The solutions for the oscillations are obtained by solving the linearized equations governing ther numerically as an initial value problem. The existence of the $r$ modes with oscillation periods approximately $1 / \mathrm{m}$ times the rotation period (where $\mathrm{m}$ is the azimuthal wave number) is confirmed, as is the confinement of the g-modes towards the equatorial regions. The results support the possibility that the coherent periodicities and the quasi-periodic behaviour seen in cataclysmic variables are caused by sets of low frequency oscillations excited in a rapidly rotating white dwarf envelope. The results of this work are to be published in full elsewhere (Papaloizou and Pringle, 1979).

\section{REFERENCES}

Papaloizou, J. and Pringle, J.E. 1978. Mon.Not.R.astr.Soc., 182, 423.

Papaloizou, J. and Pringle, J.E. 1979. Mon.Not.R.astr.Soc., in press. 